\title{
La incorporación del aprendizaje-servicio en la universidad: la experiencia de la Universidad de Barcelona
}

\author{
Mireia Esparza, Victoria Morín y Laura Rubio
}

Universidad de Barcelona

\section{Resumen}

Este artículo presenta el proceso de introducción y extensión del aprendizaje-servicio en la Universidad de Barcelona fundamentado en dos ejes que se complementan entre sí. Primero, el trabajo a nivel de universidad, coordinado por el Grupo ApS(UB) que está formado por profesorado de diferentes facultades que trabaja para su promoción y reconocimiento institucional. $Y$ en segundo lugar, la tarea que se realiza en el seno de cada facultad, a través de diferentes modelos, con la voluntad de implementar el aprendizaje-servicio en las diferentes áreas y disciplinas. Desde ambas líneas de acción se entiende el aprendizaje-servicio como una propuesta transversal a las diferentes misiones de la universidad: docencia, investigación y transferencia, desde una perspectiva de responsabilidad social. Sin tratarse de la única estrategia posible, la revisión de este proceso aporta criterios y actuaciones para la extensión del aprendizaje-servicio en la universidad.

\section{Palabras clave}

Aprendizaje-servicio, universidad, responsabilidad social universitaria. 


\title{
The incorporation of service-learning in the university: the experience of the University of Barcelona
}

\begin{abstract}
This article offers an account of how service-learning was introduced and rolled out at the University of Barcelona, focusing on two interlinked aspects. The first one is the work carried out at university level under the helm of the Service-Learning Group(UB), which is made up of academics from the various faculties who work towards promoting service-learning and granting it institutional recognition. The second aspect is the work carried out within each faculty, through different models, which seek to implement service-learning across all areas and disciplines. Work at both levels views service-learning as a cross-cutting proposal to achieve the university's main missions - teaching, research and transference- whilst adopting a social responsibility perspective. Acknowledging that service-learning is not the only possible strategy, the review offered in our paper does provide criteria and actions that justify the expansion service-learning at university level.
\end{abstract}

\section{Keywords}

Service-learning, university, university social responsability. 


\section{Introducción}

A las dos grandes misiones que tradicionalmente han definido la función de la universidad, la docencia y la investigación, desde hace unos años se les ha sumado una tercera misión, la transferencia de conocimiento, que en ciertos ámbitos se asocia a la responsabilidad social de la universidad y su contribución al desarrollo del entorno. Pero el hecho de considerar la responsabilidad social únicamente como parte de esta tercera misión parece desvincularla de la docencia y la investigación, cuando debería ser el marco general que las englobe, el eje transversal que de sentido y permita avanzar hacia los grandes retos actuales de la universidad.

El aprendizaje-servicio es un proceso que permite vincular de manera evidente y efectiva la docencia universitaria con la responsabilidad social, contribuyendo al desarrollo del compromiso del estudiante con su entorno, con el fin de mejorarlo. En este aspecto, uno de los puntos a desarrollar es la formación ciudadana de las y los estudiantes, en aras de formar profesionales competentes que sean y actúen también como ciudadanos comprometidos con la sociedad. Por otro lado, también implica el contacto directo del profesorado con las necesidades de la sociedad, fortaleciendo los vínculos entre el mundo académico, las administraciones y los agentes sociales, y favoreciendo así la llamada investigación con y para la comunidad. De este modo, el aprendizaje-servicio introduce la responsabilidad social en todos los ámbitos universitarios, transformando la universidad como una institución éticamente responsable.

El aprendizaje-servicio en la universidad puede incorporarse en distintos espacios: en asignaturas obligatorias u optativas, vinculando la propuesta a algunos de sus objetivos de aprendizaje; en prácticas curriculares y extracurriculares, aprovechando la vertiente aplicada de este espacio; en Trabajos de Final de Grado y de Trabajos de Final de Máster, ofreciendo la posibilidad de realizar investigaciones o generar productos con componente social; y, en créditos de libre elección, conectando a las y los estudiantes de diferentes cursos o estudios de una facultad con entidades sociales. Pese a que originariamente han sido los grados del ámbito más social o educativo los pioneros en incorporar el aprendizaje-servicio, cada vez son más los casos en que las áreas científicas integran esta propuesta pedagógica.

En el ámbito nacional e internacional las experiencias exitosas de aprendizaje-servicio en el ámbito universitario son numerosas. A nivel internacional las experiencias con una mayor tradición son las americanas, no en vano los trabajos de John Dewey (1967) sobre el aprendizaje activo y significativo constituyen la base teórica inicial en la que se funda el aprendizaje-servicio. Así, varias universidades de los Estados Unidos han incorporado programas de aprendizaje-servicio como estrategia institucional, pasando a ser un requerimiento en el currículum de los estudiantes universitarios. La mayoría

Esparza, M.; Morín, V. y Rubio, L. (2018). La incorporación del aprendizaje-servicio en la universidad: la experiencia de la Universidad de Barcelona. RIDAS, Revista Iberoamericana de Aprendizaje Servicio, 6, 97-114. DOI10.1344/RIDAS2018.6.10 
de ellas se agrupan desde 1985 en el Campus Compacti, creado con el ánimo de fortalecer los valores democráticos del país a través del vínculo de los estudiantes universitarios con la comunidad durante su formación. También las universidades latinoamericanas llevan años implementando programas de aprendizaje-servicio y en algunos casos como requisito para la graduación de sus estudiantes. Los inicios del aprendizaje-servicio en América Latina se remontan a 1975, cuando en Costa Rica se estableció como requisito para la graduación la realización de 300 horas de trabajo comunal universitario relacionado con el perfil profesional del estudiante (Tapia, 2017). A lo largo de los años este tipo de propuestas se han ido extendiendo a otros países y varias asociaciones han ido promoviendo las prácticas de aprendizaje-servicio en el contexto universitario. Así, en Argentina destaca la labor del Centro Latinoamericano de Aprendizaje y Servicio Solidario (CLAYSS) que, junto a colaboradores de Uruguay y Brasil, desarrolla diversas actividades no solo en América Latina, sino también en otras regiones del mundoii.

En Cataluña cabe destacar la Universidad Rovira i Virgili (URV) de Tarragona, la primera en institucionalizar el aprendizaje-servicio, incorporándolo en su Plan Estratégico de Docencia y el de la Tercera Misión, como uno de los ejes que definen esta Universidadiii. En coherencia con ello, el equipo directivo de la URV contempla como acción de gobierno el fomento del aprendizaje-servicio como actividad de formación integral de sus estudiantes.
Otras universidades, tanto catalanas como del resto del estado español, están actualmente en procesos de conseguir esta institucionalización, como una vía no solo de extensión del aprendizaje-servicio, sino también como un modo de incorporar la responsabilidad social como eje vertebrador de la universidad.

En este artículo analizaremos, partiendo de la experiencia de la Universidad de Barcelona, qué elementos son necesarios para introducir y extender el aprendizajeservicio en el ámbito universitario con éxito. A pesar de que éste no es el único modelo posible de implementación del aprendizajeservicio en la universidadiv, su revisión pone de relieve elementos clave para su desarrollo tanto a nivel institucional como a nivel de centros.

Comprobaremos la importancia de establecer redes de trabajo para facilitar este proceso de implementación, extensión y mejora. Finalmente estableceremos, desde nuestra experiencia, los retos que deben afrontar el aprendizaje-servicio en el ámbito universitario y los cambios y mejoras que deben darse para lograr estos retos, manteniendo el criterio de máxima calidad de los proyectos.

\section{El proceso de incorporación y extensión en el conjunto de la universidad}

En la Universidad de Barcelona, a diferencia de otras universidades, el aprendizaje-servicio como propuesta formativa surge desde la base, impulsada por parte de un equipo creciente de profesorado que comparte

Esparza, M.; Morín, V. y Rubio, L. (2018). La incorporación del aprendizaje-servicio en la universidad: la experiencia de la Universidad de Barcelona. RIDAS, Revista Iberoamericana de Aprendizaje Servicio, 6, 97-114. DOI10.1344/RIDAS2018.6.10 
el interés por desarrollar experiencias que conecten la universidad y la sociedad desde un marco de colaboración mutua.

El primer núcleo se sitúa como tal en la Facultad de Educación, que posteriormente tomó contacto con el programa de Clínicas Jurídicas de la Facultad de Derecho y otras experiencias en el ámbito de Salud, Filología y Economía próximas a la filosofía del aprendizaje-servicio. Es en este marco en el que, identificando algunas personas interesadas en la propuesta desde diferentes facultades, en 2013 se configura el Grupo ApS(UB), que se formaliza como grupo de trabajo del Instituto de Ciencias de la Educación (ICE) en 2016.

\subsection{El grupo ApS(UB)}

El grupo ApS(UB) tiene un carácter totalmente interdisciplinario, ya que lo integra profesorado de diversas facultades y áreas de conocimiento. Actualmente lo forman las facultades de Bellas Artes, Biblioteconomía y Documentación, Biología, Ciencias de la Tierra, Derecho, Economía y Empresa, Educación, Enfermería, Farmacia y Ciencias de la Alimentación, Filología, Geografía e Historia, Medicina, Psicología, Química y Trabajo Social.

La voluntad de este grupo ha sido, desde su inicio, difundir y extender el aprendizaje-servicio en la Universidad de Barcelona. Esta finalidad se concreta en los objetivos siguientes:

- Identificar proyectos cercanos a la idea de aprendizaje-servicio que se llevan a cabo en la Universidad de
Barcelona y que, a pesar de no haber nacido bajo esta propuesta, comparten su filosofía y componentes principales

- Dar a conocer y promover los proyectos de aprendizaje-servicio como una propuesta que puede atender de manera integradora las tres misiones de la universidad $y$, además, hacerlo desde una perspectiva alineada con la responsabilidad social universitaria

- Desarrollar proyectos de aprendizaje-servicio compartidos que permitan trabajar de manera transversal y entre facultades

- Velar por la calidad de las experiencias de aprendizaje-servicio

- Trabajar por el reconocimiento institucional

Debido a la dimensión de la universidad, el Grupo ApS(UB) ha optado por estructurarse a partir de un grupo de coordinadores de facultad, que se reúne de manera mensual para compartir su tarea, así como para dibujar y establecer las estrategias de acción compartidas a nivel de universidad.

\subsection{Líneas de trabajo y acciones a nivel de Universidad}

El Grupo ApS(UB) desarrolla su acción a través de diferentes líneas de trabajo y tareas para llevar a cabo su cometido. A continuación se apuntan algunas de las más destacadas a lo largo de estos años. 


\section{Definición y diagnóstico}

Una primera línea de trabajo ha sido, y continúa siendo, la elaboración del marco de referencia (a nivel teórico y práctico) consensuado entre los diferentes miembros del equipo sobre cómo se entiende el aprendizajeservicio y se vincula a las misiones propias de la universidad. Esta línea de trabajo se concreta en las siguientes acciones:

- Discusión y elaboración de los documentos que establecen el marco de referencia y se pueden utilizar a modo de carta de presentación

- Lectura y análisis de la documentación y las experiencias que puedan dar mayor consistencia al aprendizaje-servicio

- Identificación de las personas interesadas y las experiencias de la Universidad de Barcelona cercanas al aprendizaje-servicio

\section{Difusión y promoción}

Esta línea de trabajo supone fundamentalmente el desarrollo de actividades para dar a conocer la propuesta desde diferentes espacios, formatos y perspectivas. En este caso, las acciones en las que se concreta esta línea de trabajo son:

- Organización de la jornada anual de aprendizaje-servicio, en colaboración con el ICE de la universidad. Hasta el momento se han desarrollado cinco jornadas en las que se ha vinculado el aprendizaje-servicio a temáticas específicas como las Prácticas y Trabajos de Fin de Grado o los
Objetivos de Desarrollo Sostenible. Estos últimos pueden ser un marco interesante para el desarrollo y la integración de los proyectos de aprendizaje-servicio desde una perspectiva interdisciplinar

- Desarrollo de una página web, apoyada también por redes sociales, que permite visibilizar el aprendizajeservicio en la Universidad de Barcelona y su concreción en las diferentes facultades

- Realización de vídeos sobre experiencias o jornadas de aprendizajeservicio en la universidad

\section{Diseño e implementación de experiencias}

El grupo destina tiempo a nivel individual y colectivo a la puesta en marcha de proyectos y experiencias concretas de aprendizaje-servicio, como

- Diseño, implementación y evaluación del proyecto transversal Compartir ideas. La Universidad va al Instituto que se realiza en colaboración con el Consorcio de Educación de Barcelona y cuenta con el apoyo del Vicerrectorado de Política Docente y el Vicerrectorado de Igualdad y Acción Social

- Análisis de las posibilidades de proyectos compartidos de carácter interdisciplinar entre dos o más estudios

\section{Reconocimiento institucional}

Otra de las líneas de trabajo tiene como objetivo velar por el reconocimiento de la propuesta a nivel institucional. En 
este sentido, el grupo actúa como interlocutor con los equipos de gobierno para reivindicar las condiciones necesarias para la correcto desarrollo del aprendizaje-servicio.

\section{Desarrollo y divulgación científica}

El grupo ApS(UB) también dedica un tiempo y espacio a la investigación e innovación docentes y a la difusión de los resultados obtenidos en distintos foros y formatos:

- Desarrollo de investigaciones sobre la propuesta docente y su impacto en los estudiantes, el entorno y el profesorado, por ejemplo, en relación a la práctica reflexiva

- Publicación de artículos o participación en libros sobre aprendizaje-servicio y su desarrollo en la universidad

- Presentación de comunicaciones o ponencias en congresos, seminarios o jornadas académicos de carácter nacional e internacional

\section{Participación y contacto con otros agentes}

Finalmente, cabe destacar como línea de trabajo propia del grupo la voluntad y la puesta en marcha de acciones que garanticen la conexión de la tarea que se realiza con otros agentes:

- Contacto con entidades para el desarrollo de proyectos de aprendizajeservicio

- Interacción con la administración territorial para llevar a cabo proyectos con un mayor impacto
- Vinculación con redes sobre la temática a nivel nacional e internacional, como la Red Catalana, la Red Española o la Red Iberoamericana.

\section{Incorporación y extensión del aprendizaje-servicio en las facultades y centros}

El Grupo ApS (UB), debido a la dimensión de la Universidad de Barcelona, a su propia tradición y a la convicción de que este tipo de proyectos debe iniciarse desde la base, se organiza fundamentalmente a nivel de facultades. Esto favorece que cada centro pueda mantener su idiosincrasia y gestionarse de manera más efectiva. De esta manera, se han generado diferentes formas de desarrollar esta tarea de coordinación.

\subsection{Modelos de coordinación en las facultades}

En estos momentos, fundamentalmente existen tres modelos distintos de coordinación del aprendizaje-servicio a nivel de facultad: la Oficina de aprendizaje-servicio, las clínicas jurídicas y la figura del coordinador/a de centro. En todos los casos, resulta importante su existencia así como también que ésta sea conocida y reconocida en el seno de la facultad.

La Oficina de aprendizaje-servicio ${ }^{\vee}$ es la estructura que coordina los proyectos de aprendizaje-servicio en la Facultad de Educación. Esta coordinación se lleva a cabo a partir de un equipo formado por dos profesoras (con una descarga de horas/créditos) y el apoyo de dos becarios. La Oficina es la

\footnotetext{
Esparza, M.; Morín, V. y Rubio, L. (2018). La incorporación del aprendizaje-servicio en la universidad: la experiencia de la Universidad de Barcelona. RIDAS, Revista Iberoamericana de Aprendizaje Servicio, 6, 97-114. DOI10.1344/RIDAS2018.6.10 
encargada de a) difundir el aprendizaje-servicio en la facultad entre profesorado y estudiantes; b) coordinar, gestionar y evaluar los programas transversales a través de un catálogo general para toda la facultad; c) apoyar al profesorado para introducir el aprendizaje-servicio en asignaturas; d) construir una red de partners con entidades sociales y educativas que ofrece sus proyectos a través de una feria al inicio de curso; e) velar por la calidad de los programas de aprendizaje-servicio; y f) investigar temas relativos con el aprendizajeservicio y su desarrollo en la facultad.

El modelo de clínicas jurídicas es el que permite el desarrollo de proyectos de aprendizaje-servicio en la Facultad de Derecho. El proyecto se conoce como "derecho al Derecho"vi y funciona mediante un equipo docente con un coordinador general y 15 clínicas con un coordinador específico para cada una de ellas. Además, el proyecto cuenta con el apoyo administrativo de la facultad. Las actividades que se desarrollan desde las clínicas son variadas: realización de prácticas, preparación de informes y talleres, asesoramiento jurídico, publicación de textos, organización de jornadas o realización de trabajos de investigación. Desde la coordinación deben garantizarse las condiciones para que estas tareas puedan llevarse a cabo.

Finalmente el modelo de coordinación de centro se está desarrollando en el resto de facultades implicadas en el Grupo ApS (UB) aunque hay diversidad en su nivel de acción y reconocimiento institucional. En la mayoría de las facultades la tarea se concreta en la figura del coordinador/a que asume las responsabilidades de la promoción del aprendizaje-servicio entre equipo decanal, profesorado y estudiantes de la facultad, así como de la gestión que se deriva del desarrollo de este tipos de proyectos en el centro. Siempre que sea posible, es importante que esta coordinación se realice por parte de un equipo, evitando que la tarea recaiga en una sola persona.

\subsection{Líneas de trabajo y acciones a nivel de facultad}

En este apartado se intenta dar una visión de conjunto de las distintas acciones que, en mayor o menor medida, se llevan a cabo en las diversas facultades como funciones de la coordinación.

Detección de posibilidades de proyectos en el marco de los estudios

Desde la coordinación de centro se realizan distintas acciones encaminadas a incorporar el aprendizaje-servicio en cada centro:

- Detección de sensibilidades entre el profesorado candidato a participar en proyectos de aprendizajeservicio. El hecho de trabajar a nivel de centro facilita la identificación de los intereses y motivaciones del profesorado, así como la comunicación y el trabajo en común con éstos.

- Identificación de asignaturas o espacios en los que sea posible trabajar a partir del aprendizaje- 
servicio. Por ejemplo, en el caso de la Facultad de Enfermería, el Trabajo de Fin de Grado ofrece un marco lo suficientemente flexible en el que trabajar en proyectos de salud comunitaria innovadores, ofreciendo a la ciudadanía algunos servicios de valor para la salud.

- Implicación del decanato en la asignación de condiciones y recursos que faciliten el desarrollo de proyectos de aprendizaje-servicio en la facultad. La aportación de los órganos de gobierno permite mantener el interés y el compromiso por parte del profesorado y viceversa.

Apoyo en el diseño de proyectos de aprendizaje-servicio

Una vez identificados e implicados profesores y asignaturas, desde la coordinación se brinda apoyo y asesoramiento en la creación e implementación de los proyectos:

- Valoración de las posibilidades de proyectos de aprendizaje-servicio en cada propuesta identificada. En este caso, pueden desarrollarse reuniones conjuntas o individuales para consensuar con el profesorado: número estudiantes participantes, estrategias de tutorización, productos a presentar o reconocimiento, entre otras.

- Asesoramiento al profesorado a lo largo del proceso de implementación de los proyectos. El coordinador/a puede solucionar dudas o acompañar en momentos concretos según las necesidades en cada caso.

\section{Vínculo con las entidades colaboradoras}

Un aspecto esencial a tener en cuenta en los proyectos de aprendizajeservicio es la relación con las entidades participantes. En ella los coordinadores pueden resultar un elemento clave mediante distintas acciones:

- Creación de una cartera de entidades interesadas en participar en proyectos de aprendizajeservicio. Es necesario hacer una proceso de búsqueda activo, especialmente al inicio de incorporación de la propuesta en la facultad. En este caso puede utilizarse internet, guías de entidades, contactos previos o propuestas de los propios estudiantes.

- Valoración del potencial de las entidades y su idoneidad en relación a los criterios propios de cada facultad y sus objetivos formativos.

- Cuidado de la relación con los referentes de las entidades a lo largo de todo el proceso a través de llamadas, visitas u otros espacios de encuentro.

\section{Velar por la calidad de proyectos}

Es imprescindible mantener la calidad en los proyectos de aprendizajeservicio y evitar el "todo vale". Para ello, desde la coordinación de centro deben tenerse en cuenta diferentes tipos de criterios:

- Los que apunta la propia 
metodología de aprendizajeservicio. Para esta tarea pueden utilizarse herramientas como la rúbrica de autoevaluación de proyectos.

- Los que señalan las necesidades de las entidades. Para ello es importante establecer espacios de contacto y reflexión con sus referentes.

- Y, finalmente, los relacionados con la percepción de aquello que es relevante por parte de los estudiantes y que incluyen desde los aprendizajes adquiridos a las condiciones necesarias para el desarrollo del proyecto.

\section{Difusión del aprendizaje-servicio}

Las acciones de difusión de los proyectos de aprendizaje-servicio dentro y fuera de las facultades es un aspecto clave para iniciarlos y consolidar su desarrollo y pueden ser variadas y complementarias.

A nivel interno, desde la coordinación se pueden organizar e informar de actividades para el profesorado y estudiantes. Por ejemplo, en la facultad de Economía y Empresa se organiza una feria de entidades en la que contactan estudiantes y diversas organizaciones con las que desarrollar proyectos de aprendizaje-servicio.

A nivel externo la promoción del aprendizaje-servicio favorece el trabajo en red entre distintas entidades, colaborando en el desarrollo de proyectos compartidos. En otro sentido, también es importante dar a conocer a la comunidad científica los proyectos que se realizan en cada centro, mediante la participación en congresos y jornadas.

\section{Las claves del trabajo}

A la luz de la revisión del sentido y el trabajo realizado por el Grupo ApS(UB) para la extensión del aprendizajeservicio en la Universidad de Barcelona podemos decir que, algunas de las principales claves de este proceso han sido:

1. Partir desde la base y priorizar el desarrollo de una masa crítica amplia de personas y experiencias propiamente de aprendizaje-servicio o próximas a la metodología desde diferentes áreas previamente a ascender la propuesta a nivel político.

2. Tener claro y compartir también desde el principio, que el verdadero desarrollo y arraigo de la propuesta supone no reducir el aprendizajeservicio a una propuesta de innovación docente y velar por su reconocimiento institucional y las condiciones que lo han de hacer posible.

3. La voluntad de establecer una organización que centre su trabajo en cada facultad, a través del coordinador de centro y que, a su vez, pretenda tener una imagen de conjunto y establecer líneas de trabajo compartidas, abriendo espacios para la progresiva incorporación de todas y todos los que estén interesados.

4. Aprovechar diferentes espacios, 
momentos y formatos para hacer presente el aprendizaje-servicio a lo largo del curso y en el conjunto de la universidad, así como también para identificar personas y experiencias afines desde donde hacer crecer el proyecto.

5. Idear proyectos compartidos que, más allá de la tarea y las experiencias propias de cada facultad, permitan desarrollar y afrontar conjuntamente las cuestiones prácticas que subyacen a un proyecto de aprendizaje-servicio.

\section{Aspiraciones y retos de futuro}

El aprendizaje-servicio como una propuesta educativa en favor de la responsabilidad social universitaria pasa por la consecución de ciertos retos y aspiraciones.

En primer lugar, consideramos necesario continuar con la promoción y consolidación del aprendizaje-servicio en todos los ámbitos de la universidad, y especialmente en aquellos en los que tradicionalmente han tenido menos presencia. El proceso de incorporación de esta propuesta debe ser constante pero pausado, para que no se convierta en algo pasajero fruto de una moda y vacío de contenido. Para ello, además de analizar las posibilidades desde las diferentes disciplinas, se debe avanzar en la vinculación del aprendizajeservicio con las diferentes misiones de la universidad, siguiendo un proceso de reflexión compartida entre los diferentes agentes implicados.

Velar por la calidad de los proyectos de aprendizaje-servicio continúa siendo un reto en sí mismo. Es necesario evaluar los resultados de los proyectos de aprendizaje-servicio, investigando y valorando su impacto en los distintos aspectos sobre los que actúa: el aprendizaje por parte de los estudiantes, el impacto sobre su nivel de compromiso cívico y profesionalización, la mejora que supone en la sociedad o la potenciación de la responsabilidad social de la institución universitaria, entre otros. Claramente queda un largo recorrido por hacer en esta dirección.

Otro aspecto en el que insistir es las posibilidades que aporta el aprendizajeservicio a nivel de investigación. Debe aprovecharse la oportunidad que supone la investigación basada en la comunidad, que tiene en cuenta las necesidades reales de ésta y genera un retorno directo. Pero, además, el aprendizaje-servicio permite también la posibilidad de incorporar a los estudiantes en este proceso, favoreciendo la mejora de sus competencias investigadoras y su profesionalización.

Un punto a tener en cuenta es también la posibilidad de establecer líneas de trabajo conjunto entre distintas facultades y universidades. Estas relaciones pueden establecerse en el marco de grupos intra-universitarios, como el grupo ApS(UB), o bien redes inter-universitarias como la Red de Aprendizaje Servicio en las universidades catalanas, la red nacional o las colaboraciones internacionales. Estas relaciones sin duda enriquecen enormemente las experiencias de 
aprendizaje-servicio de cada centro, permitiendo avanzar hacia un nivel de máxima excelencia de los proyectos.

Finalmente consideramos que pese a que en la Universidad de Barcelona la introducción y desarrollo del aprendizaje-servicio ha sido un proceso iniciado desde la base, para que el profesorado continúe vinculado es necesario que su labor se vea reconocida a nivel institucional. Un proyecto de aprendizaje-servicio suele suponer un trabajo extra para el profesor que debe ser reconocido, ya sea a nivel de méritos docentes o bien con un mayor número de horas asignadas a las asignaturas que sigan esta metodología. También son necesarias condiciones para favorecer las relaciones con las entidades del entorno y propiciar proyectos interdisciplinares. Es importante, por lo tanto, implicar a los equipos rectorales en el reconocimiento del aprendizajeservicio desde una perspectiva poliédrica que incluya las diferentes misiones, que sea respetuosa con los proyectos vigentes y que aporte las condiciones necesarias para favorecer la consolidación y extensión de este tipo de proyectos.

\section{Referencias bibliográficas}

Dewey, J. (1967). Experiencia y Educación. Buenos Aires: Losada.

Tapia, M.N (2017). Aprendizajeservicio, un movimiento pedagógico mundial. En: Rubio, L. y Escofet, A. (Coords.) Aprendizaje-servicio (ApS): claves para su desarrollo en la universidad. Barcelona: Octaedro/ICEUB.

\footnotetext{
i Para mayor información, revisar https://compact.org

ii Para mayor información, revisar http://www.clayss.org

iii Estos documentos pueden descargarse en la página web sobre el aprendizaje-servicio de la URV http://www.urv.cat/ca/estudis/modeldocent/aprenentatge-servei

iv Una de las propuestas más conocidas en este sentido es la rúbrica para la institucionalización del aprendizaje servicio en la educación superior. En 2003 se desarrolló una revisión y traducción de la misma http://educaciocp89.webjoomla.es/wp-content/uploads/04Furco-2-Castellano.pdf

v Para mayor información, puede verse la página web de Oficina de aprendizaje-servicio de la Facultad de Educación en https://www.ub.edu/portal/web/educacio/aps vi Para mayor información, puede verse la página web del proyecto "derecho al Derecho" de la Facultad de Derecho en http://www.ub.edu/dretaldret
} 